\title{
Mismatch repair deficiency commonly precedes adenoma formation in Lynch Syndrome-Associated colorectal tumorigenesis
}

Shigeki Sekine ${ }^{1,2,3}$, Taisuke Mori ${ }^{1,2,3}$, Reiko Ogawa ${ }^{2}$, Masahiro Tanaka ${ }^{1}$, Hiroshi Yoshida ${ }^{1}$, Hirokazu Taniguchi ${ }^{1}$, Takeshi Nakajima ${ }^{3,4}$, Kokichi Sugano ${ }^{3,5}$, Teruhiko Yoshida ${ }^{3,6}$, Mamoru Kato ${ }^{7}$, Eisaku Furukawa ${ }^{7}$, Atsushi Ochiai ${ }^{8}$ and Nobuyoshi Hiraoka ${ }^{1,2,3}$

${ }^{1}$ Division of Pathology and Clinical Laboratories, National Cancer Center Hospital, Tokyo, Japan; ${ }^{2}$ Division of Molecular Pathology, National Cancer Center Research Institute, Tokyo, Japan; ${ }^{3}$ Department of Genetic Medicine and Services, National Cancer Center Hospital, Tokyo, Japan; ${ }^{4}$ Division of Endoscopy, National Cancer Center Hospital, Tokyo, Japan; ${ }^{5}$ Oncogene Research Unit/Cancer Prevention Unit, Tochigi Cancer Center Research Institute, Tochigi, Japan; ${ }^{6}$ Division of Genetics, National Cancer Center Research Institute, Tokyo, Japan; ${ }^{7}$ Department of Bioinformatics, National Cancer Center Research Institute, Tokyo, Japan and ${ }^{8}$ Division of Pathology, Research Center for Innovative Oncology, National Cancer Center, Kashiwa, Chiba, Japan

Lynch syndrome is a cancer predisposition syndrome caused by germline mutations in mismatch repair (MMR) genes. MMR deficiency is a ubiquitous feature of Lynch syndrome-associated colorectal adenocarcinomas; however, it remains unclear when the MMR-deficient phenotype is acquired during tumorigenesis. To probe this issue, the present study examined genetic alterations and MMR statuses in Lynch syndrome-associated colorectal adenomas and adenocarcinomas, in comparison with sporadic adenomas. Among the Lynch syndrome-associated colorectal tumors, 68 of 86 adenomas $(79 \%)$ and all adenocarcinomas were MMR-deficient, whereas all the sporadic adenomas were MMR-proficient, as determined by microsatellite instability testing and immunohistochemistry for MMR proteins. Sequencing analyses identified APC or CTNNB1 mutations in the majority of sporadic adenomas $(58 / 84,69 \%)$ and MMR-proficient Lynch syndrome-associated adenomas $(13 / 18$, 72\%). However, MMR-deficient Lynch syndrome-associated adenomas had less APC or CTNNB1 mutations (25/68, 37\%) and frequent frameshift RNF43 mutations involving mononucleotide repeats (45/68, 66\%). Furthermore, frameshift mutations affecting repeat sequences constituted 14 of 26 APC mutations (54\%) in MMR-deficient adenomas whereas these frameshift mutations were rare in MMR-proficient adenomas in patients with Lynch syndrome $(1 / 12,8 \%)$ and in sporadic adenomas $(3 / 52,6 \%)$. Lynch syndrome-associated adenocarcinomas exhibited mutation profiles similar to those of MMR-deficient adenomas. Considering that WNT pathway activation sufficiently drives colorectal adenoma formation, the distinct mutation profiles of WNT pathway genes in Lynch syndrome-associated adenomas suggest that MMR deficiency commonly precedes adenoma formation.

Modern Pathology (2017) 30, 1144-1151; doi:10.1038/modpathol.2017.39; published online 26 May 2017

Lynch syndrome is one of the most common inherited cancer predisposition syndromes, and is caused by germline mutations in mismatch repair (MMR) genes, including MLH1, MSH2, PMS2, and

Correspondence: Dr S Sekine, MD, PhD Division of Pathology and Clinical Laboratories, National Cancer Center Hospital, 5-1-1, Tsukiji, Chuo-ku, Tokyo 104-0045, Japan.

E-mail: ssekine@ncc.go.jp

Received 24 January 2017; revised 28 March 2017; accepted 29 March 2017; published online 26 May 2017
MSH6. ${ }^{1,2}$ Patients with Lynch syndrome have increased risks for cancers of multiple organs, including the colon, stomach, small intestine, and urinary bladder, with colorectal cancer being the most prevalent. ${ }^{3,4}$ Although both Lynch syndromeassociated and sporadic colorectal cancers predominantly develop through the adenoma-carcinoma pathway, tumorigenesis in these two different clinical settings involves different sets of molecular abnormalities. ${ }^{1,2}$ Most importantly, MMR deficiency resulting from the biallelic inactivation of MMR 
genes is a consistent feature of Lynch syndromeassociated colorectal cancers, but does not play a role in the conventional pathway of sporadic tumorigenesis. ${ }^{5-10}$

MMR deficiency is a virtually ubiquitous feature of advanced adenomas and adenocarcinomas associated with Lynch syndrome, whereas a subset of small, lowgrade adenomas is MMR-proficient. ${ }^{6-10}$ These observations led to the suggestion that MMR deficiency is acquired after adenoma formation during colorectal tumorigenesis in Lynch syndrome. ${ }^{2,9}$ However, a recent study identified MMR-deficient crypt foci, characterized by MMR protein loss and microsatellite instability in the absence of adenomatous morphology, as a potential precursor of colorectal tumors in patients with Lynch syndrome. ${ }^{11}$ This implies that MMR protein loss might precede adenoma formation at least in some instances.

Activation of the WNT signaling pathway due to $A P C$ or CTNNB1 mutations is the most common molecular abnormality in sporadic colorectal cancers. ${ }^{12-14}$ These genetic alterations are regarded as the initial molecular event in sporadic colorectal carcinogenesis via the conventional adenoma-carcinoma pathway and are sufficient to induce adenoma formation by themselves. ${ }^{15-18}$ However, the prevalence of $A P C$ mutations in Lynch syndrome-associated adenocarcinomas is significantly lower than that in sporadic cases. ${ }^{19}$ RNF43 is another recently identified tumor suppressor gene related to WNT signaling. ${ }^{20}$ RNF43 is a transmembrane E3 ligase that ubiquitinates and downregulates WNT receptors, Frizzled and LRP; therefore, RNF43 inactivation potentiates WNT signaling by enhancing ligand-dependent activation of this pathway. ${ }^{21} R N F 43$ has two mononucleotide repeats in its coding sequence and frameshift mutations affecting these repeat sequences are frequent in sporadic MMR-deficient colorectal cancers, ${ }^{20}$ however, their involvement in Lynch syndrome-associated tumorigenesis remains elusive.
The present study aimed to characterize the relationship between MMR deficiency and genetic alterations in colorectal tumorigenesis in Lynch syndrome. We examined genetic alterations involved in early colorectal tumorigenesis, including those related to the WNT signaling pathway, and MMR statuses in Lynch syndrome-associated colorectal adenomas and adenocarcinomas in comparison with sporadic colorectal adenomas.

\section{Materials and methods}

\section{Cases}

This study was approved by the Ethics Committee of the National Cancer Center, Tokyo, Japan. In the present study, we analyzed 86 adenomas and 36 adenocarcinomas of the colorectum obtained from 44 patients with genetically confirmed Lynch syndrome (Table 1 and Supplementary Table 1). Among these, 62 adenomas and 10 adenocarcinomas were analyzed for MMR protein expression in our previous study. ${ }^{10}$ Lesions included in the present study were selected based on the availability of sufficient tissue samples for DNA extraction. Newly added cases were selected by the availability of small adenomas or adenocarcinomas to increase the variability of lesions to be analyzed. In addition, 84 sporadic colorectal tubular adenomas were analyzed for comparison. All tissue samples were obtained by endoscopic or surgical resection at the National Cancer Center Hospital, Tokyo, Japan, fixed in $10 \%$ formalin and embedded in paraffin. Histological diagnoses of the lesions were made based on the World Health Organization classification. All the germline mutations in patients with Lynch syndrome were categorized as classes IV or $\mathrm{V}$ by InSiGHT classification. ${ }^{22}$

Table 1 Summary of Lynch syndrome-associated lesions

\begin{tabular}{|c|c|c|c|c|c|c|}
\hline & & Adenoma $(\mathrm{n}=86)$ & & & Adenocarcinoma $(\mathrm{n}=36)$ & \\
\hline Age, year-old, median (range) & & $62(34-84)$ & & & $51(20-74)$ & \\
\hline \multicolumn{7}{|l|}{ Sex } \\
\hline Male & & 45 & & & 16 & \\
\hline Female & & 41 & & & 20 & \\
\hline \multicolumn{7}{|l|}{ Location } \\
\hline Proximal & & 34 & & & 22 & \\
\hline Distal & & 52 & & & 14 & \\
\hline \multirow[t]{4}{*}{ Polyp size, mm/T factor } & $\leq 5$ & & 32 & T1 & & 8 \\
\hline & $6-10$ & & 31 & $\mathrm{~T} 2$ & & 8 \\
\hline & $>10$ & & 23 & T3 & & 18 \\
\hline & & & & $\mathrm{T} 4$ & & 2 \\
\hline \multicolumn{7}{|l|}{ Germline mutation } \\
\hline MLH1 & & 25 & & & 11 & \\
\hline MSH2 & & 60 & & & 24 & \\
\hline MSH6 & & 1 & & & 1 & \\
\hline
\end{tabular}

Proximal colon, cecum to transverse colon; Distal colon, descending colon to rectum. 


\section{Immunohistochemistry}

Immunohistochemical analysis was performed on formalin-fixed paraffin-embedded specimens, as described previously. ${ }^{10}$ Antigen retrieval was performed by autoclaving in $10 \mathrm{mM}$ citrate buffer $(\mathrm{pH}$ 6.0) for $10 \mathrm{~min}$. Primary antibodies used were antiMLH1 (ES05, 1:200 dilution; Dako, Glostrup, Denmark), anti-MSH2 (FE11, 1:200 dilution; Calbiochem, La Jolla, CA, USA), anti-PMS2 (A16-4, 1:200 dilution; Biocare Medical, Concord, CA, USA), and anti-MSH6 antibodies (SP93, 1:200 dilution; Spring Bioscience, Pleasanton, CA, USA). We used an automated stainer (Dako) and EnVision Detection System (Dako) according to the vendor's protocol. Normal colonic epithelial and stromal cells served as internal positive controls. Tumors showing significantly reduced or loss of expression of any MMR proteins were deemed to be MMR-deficient, and those retaining all four MMR proteins were regarded as MMR-proficient.

\section{DNA Extraction}

Deparaffinized 10- $\mu$ m-thick sections from each paraffin block were microdissected using sterilized toothpicks under a microscope to enrich tumor content. The microdissected samples were subjected to DNA extraction using the QIAamp DNA FFPE Tissue Kit (Qiagen, Hilden, Germany).

\section{Microsatellite Instability Testing}

Microsatellite instability testing was performed using five mononucleotide repeat markers, as described previously. ${ }^{23}$ Tumors in which two or more of the five markers exhibited instability were regarded as microsatellite instability-high.

\section{Next-Generation Sequencing}

Next-generation sequencing libraries were prepared by two-step tailed PCR. ${ }^{24}$ The first round PCR was performed using two pools of primers consisting of a gene-specific sequence and a consensus primerbinding sequence. Each pool contains 12 pairs of primers targeting frequently mutated regions of $A P C$, BRAF, CTNNB1, KRAS, NRAS, and RNF43 (Supplementary Table 2). Each PCR was performed using 10 ng genomic DNA with Kapa HiFi Hotstart Ready Mix (Kapa Biosystems, Boston, MA, USA). Amplification conditions were as follows: initial denaturation for $3 \mathrm{~min}$ at $95^{\circ} \mathrm{C}, 23$ cycles of $20 \mathrm{~s}$ at $98^{\circ} \mathrm{C}, 20 \mathrm{~s}$ at $64^{\circ} \mathrm{C}$, and $20 \mathrm{~s}$ at $72^{\circ} \mathrm{C}$, and final extension of $3 \mathrm{~min}$ at $72^{\circ} \mathrm{C}$. The PCR products were purified using the Agencourt AMPure XP kit (Beckman Coulter, Brea, CA, USA) and eluted in $30 \mu \mathrm{l}$ low TE buffer.

The second round PCR was performed using $5 \mu \mathrm{l}$ of purified first round PCR products to incorporate sample-specific indexes and P5/P7 flow-cell binding sequences (Supplementary Table 2). Amplification conditions of the second round PCR were as follows: initial denaturation for $3 \mathrm{~min}$ at $95^{\circ} \mathrm{C}, 10$ cycles of $20 \mathrm{~s}$ at $98^{\circ} \mathrm{C}, 20 \mathrm{~s}$ at $65^{\circ} \mathrm{C}$, and $20 \mathrm{~s}$ at $72^{\circ} \mathrm{C}$, and final extension of $3 \mathrm{~min}$ at $72^{\circ} \mathrm{C}$. The products were purified and eluted in $30 \mu \mathrm{l}$ low TE buffer as mentioned above.

Sequencing was performed by using MiSeq (Illumina, San Diego, CA, USA) and MiSeq Reagent Kit v3 (150 cycles) (Illumina) according to the manufacturer's protocol. The resulting sequences were mapped onto the human reference genome hg19, following removal of primer sequences on each end of the amplicons by using TruSeq Amplicon application (Illumina). Sequence variations with variant allele frequencies of more than $10 \%$ were identified as candidate mutations. Synonymous mutations and common single nucleotide polymorphism, based on the Single Nucleotide Polymorphism Database build 137, were excluded. A subset of mutations detected by next-generation sequencing was subjected to Sanger sequencing for confirmation. The primers and methods for Sanger sequencing were, as described previously. ${ }^{25}$

\section{Statistical Analysis}

Fisher's exact test was used to analyze each 2-by-2 table. $P$ values $<0.05$ were considered to indicate statistical significance.

\section{Results}

Immunohistochemically, 68 adenomas and all the adenocarcinomas from patients with Lynch syndrome showed a homogeneous loss of at least one MMR protein and were deemed MMR-deficient (Figure 1a and Supplementary Table 1). The MMR proteins lost were always consistent with the underlying germline mutation. The remaining 18 Lynch syndrome-associated adenomas and all the sporadic adenomas retained all four MMR proteins, and were regarded as MMR-proficient. Lynch syndromeassociated adenomas and adenocarcinomas underwent further microsatellite instability testing. Consistent with the results of immunohistochemistry, all the MMR-deficient adenomas and adenocarcinomas were microsatellite instability-high, and all the adenomas with intact MMR protein expression were microsatellite-stable (Figure $1 \mathrm{~b}$ and Supplementary Table 1). At least one of the tumors in each patients with Lynch syndrome was MMR-deficient, supporting the diagnosis of Lynch syndrome.

Next-generation sequencing resulted in the median total coverage per sample of 272,666 reads (range, 97301 to 391887 ). Median coverage per amplicon across the all samples was 8182 reads (range, 128 to 97 301). After exclusion of synonymous variants and common single nucleotide polymorphism, 238 non- 
a

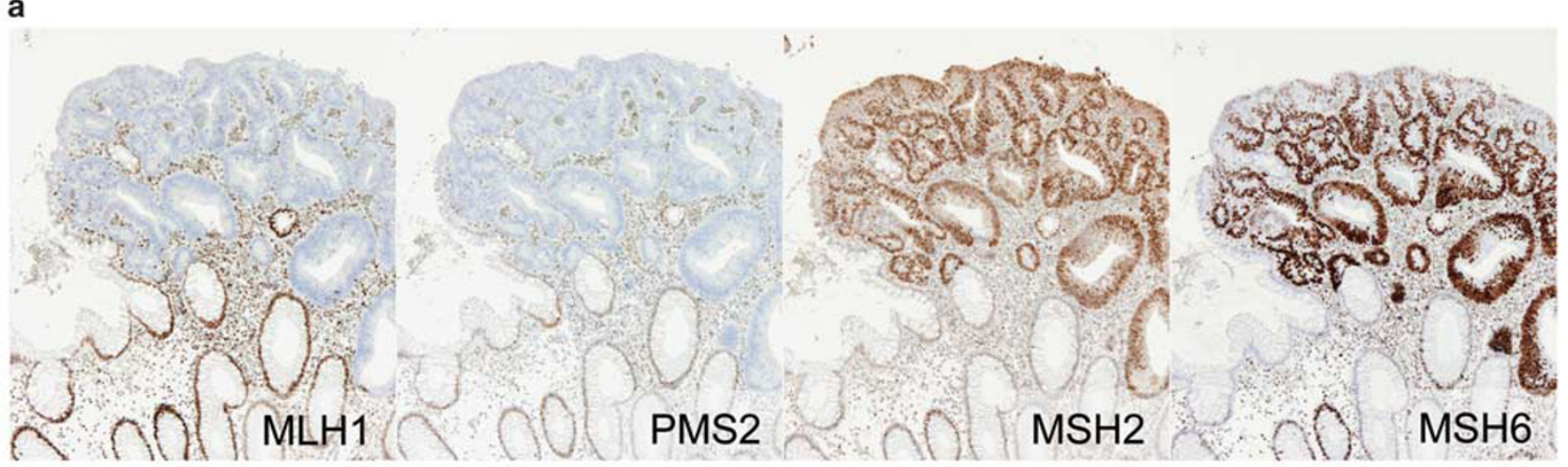

b
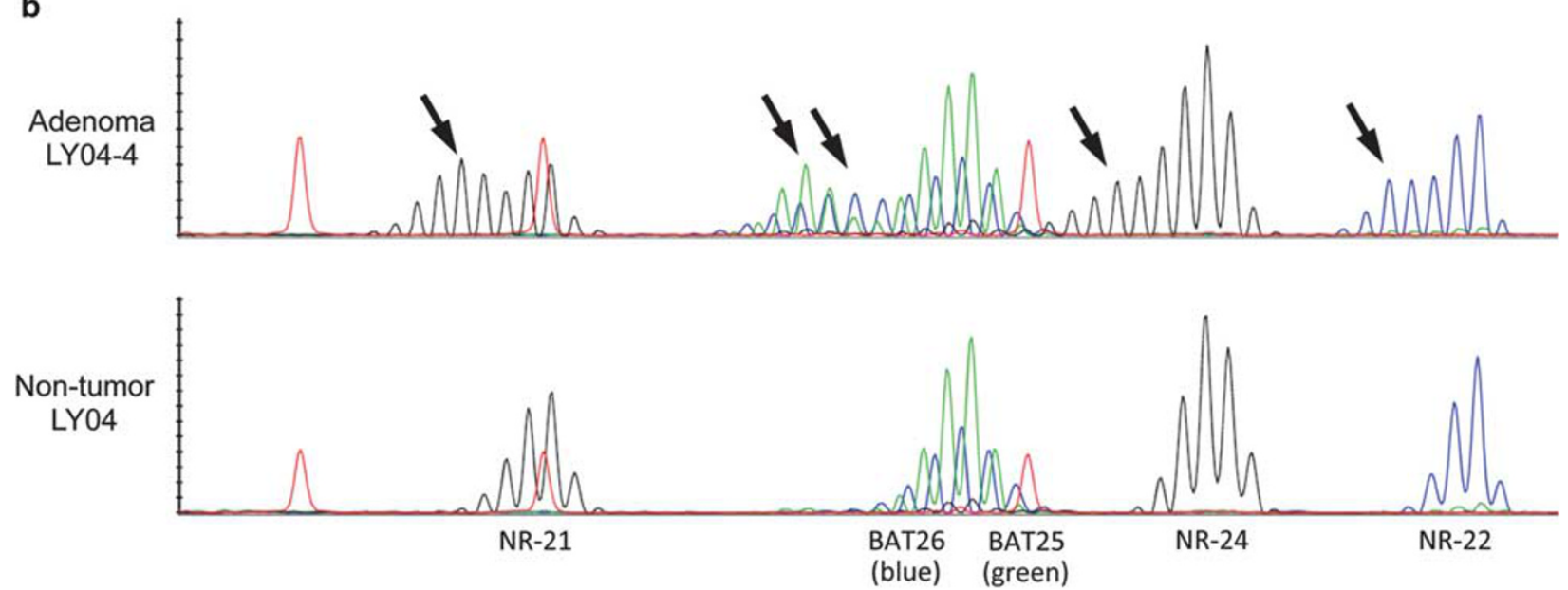

Figure 1 Immunohistochemistry for mismatch repair (MMR) proteins and microsatellite instability testing. (a) Immunohistochemical analysis of a Lynch syndrome-associated adenoma, showing loss of MLH1 and PMS2 and retention of MSH2 and MSH6 expression. (b) Microsatellite instability testing using five mononucleotide repeat markers. All the five markers show instability in this adenoma sample (arrows). Red peaks represent size markers.

synonymous variants were identified (Figure 2 and Supplementary Table 1). We analyzed 102 of the detected mutations by Sanger sequencing and confirmed all of them. For APC mutations, only nonsense and frameshift mutations were included in the following analyses, as most missense mutations are considered non-pathogenic. ${ }^{26}$

Protein-truncating APC mutations were significantly less frequent in Lynch syndrome-associated adenomas $(34 / 86,40 \%)$ than in sporadic adenomas (50/84, 60\%; $P=0.014)$. CTNNB1 mutations were present in minor subsets in both Lynch syndromeassociated $(4 / 86,5 \%)$ and sporadic adenomas (8/84, $10 \%)$. RNF43 was exclusively and frequently altered in Lynch syndrome-associated adenomas (45/86, $52 \%)$. KRAS, NRAS, and BRAF mutations were uncommon in both groups of adenomas. Adenocarcinomas in patients with Lynch syndrome frequently had RNF43 mutations similar to adenomas (20/36, $56 \%)$, and in addition, KRAS mutations (16/36, $44 \%)$. APC mutations were infrequent (10/36, 28\%).

Lynch syndrome-associated adenomas showed clearly different mutation profiles depending on the MMR statuses. RNF43 mutations were exclusive to MMR-deficient adenomas (45/68, 66\%; Figure 3a) and were mostly frameshift mutations affecting mononucleotide repeats. APC or CTNNB1 mutations were more frequent in MMR-proficient adenomas, compared with MMR-deficient adenomas (13/18, 72 vs $25 / 68,37 \% ; P=0.0086)$. Furthermore, MMRdeficient adenomas showed a characteristic $A P C$ mutation spectrum. The mutation cluster region of $A P C$ contains short repeat sequences: an A5-repeat at codon 1455, an AG5-repeat at codon 1465, and an A6-repeat at codon 1554. More than half of $A P C$ mutations in MMR-deficient adenomas were insertions or deletions involving these repeat sequences (14/26, 54\%; Figures 3b and c). These frameshift mutations were also common in Lynch syndromeassociated adenocarcinomas $(6 / 11,55 \% ; P=1.0)$, but were significantly less frequent in Lynch syndromeassociated MMR-proficient adenomas (1/12, 8\%; $P=0.0080)$ and in sporadic adenomas $(3 / 52,6 \%$; $\left.P=3.6 \times 10^{-6}\right)$. RNF43 mutations often coexisted with $A P C$ or CTNNB1 mutations in Lynch syndrome-associated adenomas and adenocarcinomas. These mutations tended to be mutually exclusive of each other, but did not show statistically 


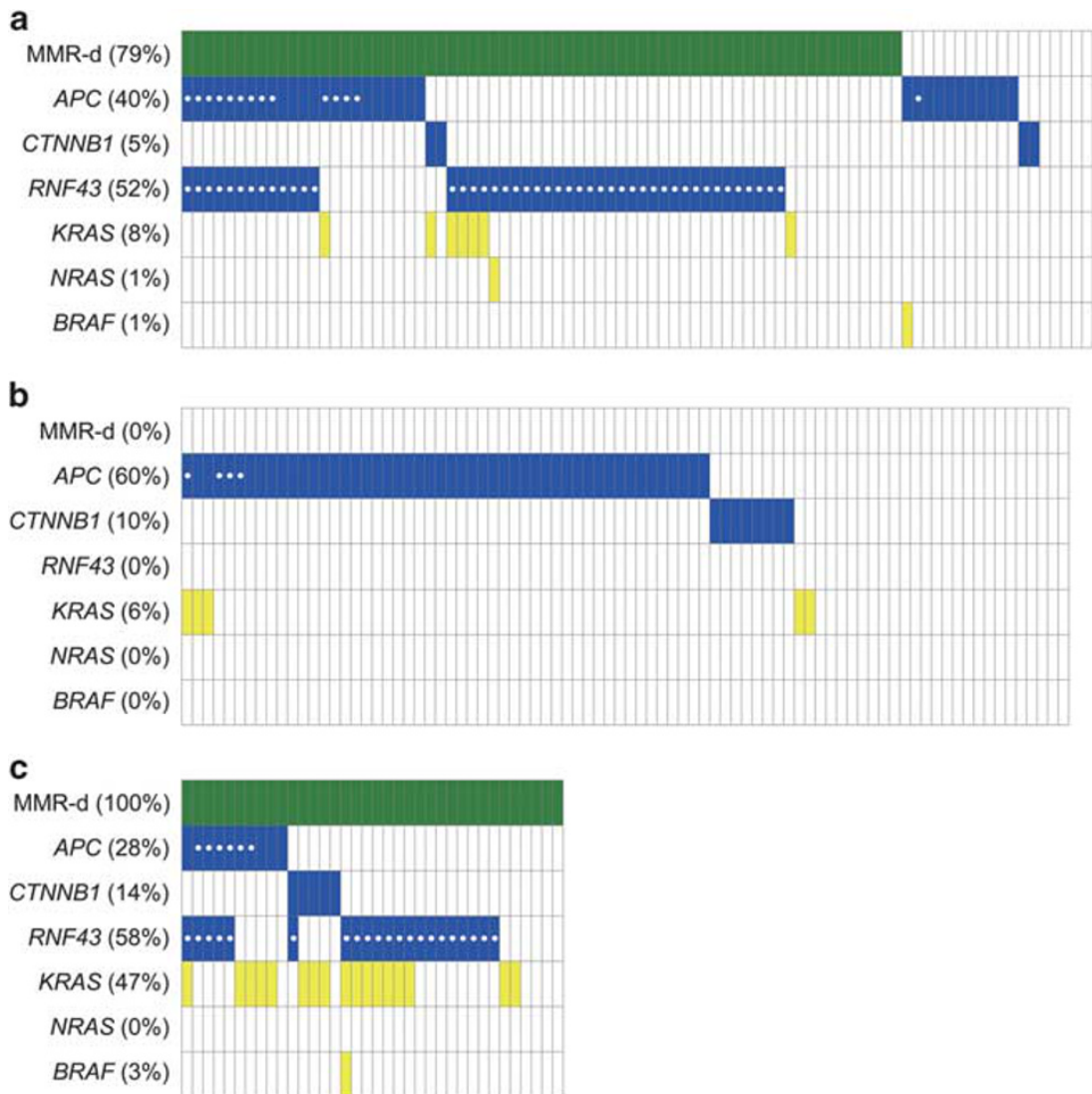

Figure 2 Mismatch repair status and genetic alterations in Lynch syndrome-associated (a) and sporadic adenomas (b) and Lynch syndrome-associated adenocarcinomas (c). The majority of Lynch syndrome-associated adenomas and all adenocarcinomas are mismatch repair-deficient (MMR-d, green), whereas all sporadic adenomas are MMR-proficient. RNF43 mutations are exclusive to MMR-deficient tumors. Among the WNT pathway genes (blue), APC and CTNNB1 mutations are always mutually exclusive, but RNF43 mutations often coexist with APC or CTNNB1 mutations. KRAS mutations are rare in Lynch syndrome-associated and sporadic adenomas but are common in Lynch syndrome-associated adenocarcinomas. Only protein-truncating mutations are indicated for APC. White circles indicate frameshift mutations affecting repeat sequences.

significant correlation (MMR-deficient adenoma, $P=0.069$; adenocarcinoma, $P=0.090)$.

\section{Discussion}

Adenoma is a major precursor of both Lynch syndrome-associated and sporadic colorectal cancers. However, the present study showed that adenomas that develop on these two different backgrounds have distinct molecular features. Consistent with previous studies, we found that MMR deficiency was common in Lynch syndrome-associated adenomas, but was absent in sporadic adenomas. ${ }^{5-10}$ In addition, our study showed that Lynch syndrome-associated adenomas had less $A P C$ mutations and frequent RNF43 mutations, indicating distinct modes of WNT pathway activation. Furthermore, Lynch syndrome-associated colorectal adenomas have clearly different genetic profiles depending on MMR status. MMR-deficient adenomas showed mutation profiles characteristic to Lynch syndrome-associated adenomas, as described above. In contrast, mutation profiles of MMR-proficient adenomas are indistinguishable from those of sporadic adenomas.

Two-thirds of Lynch syndrome-associated MMRdeficient adenomas had frameshift RNF43 mutations affecting mononucleotide repeats. Furthermore, APC mutations in Lynch syndrome-associated MMRdeficient adenomas were predominantly frameshift mutations affecting repeat sequences, which were rare in Lynch syndrome-associated MMR-proficient adenomas and sporadic adenomas. Increased prevalence of frameshift mutations is consistent with the consequence of MMR deficiency; in fact, these RNF43 and APC mutation patterns have been reported to be also common in sporadic MMRdeficient colorectal cancers. ${ }^{20,27}$

Considering that a subset of small, low-grade Lynch syndrome-associated adenomas is MMR-proficient, ${ }^{6-10}$ MMR deficiency has been thought to be acquired during the progression from early to advanced adenomas. ${ }^{2,9}$ However, this model contradicts the present results. As WNT signaling pathway activation sufficiently drives adenoma formation, ${ }^{15,16}$ the characteristic mutation spectra of RNF43 and APC in 


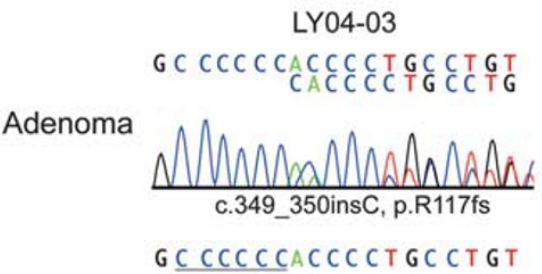

Non-tumor

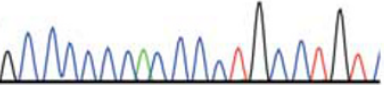

LY01-01

AAGGCGGGGGGGTCCCTC C G

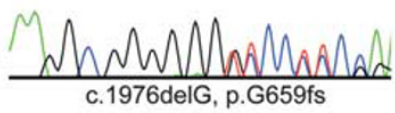

AAGGCGGGGGGGTCCCTCCG

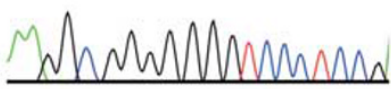

b

LY03-4

LY17-3

LY24-5

Adenoma
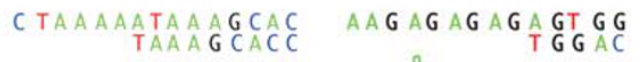

G AAAAAACTATTGAT
ACTA TTGA
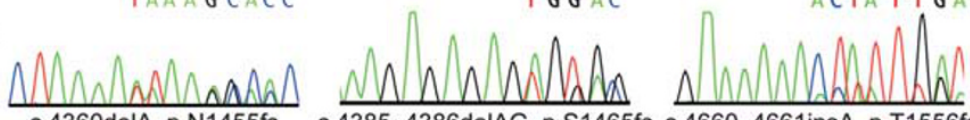

c.4360delA, p.N1455fs c.4385_4386delAG, p.S1465fs c.4660_4661insA, p.T1556fs

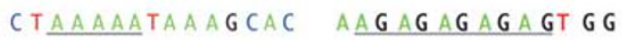

GAAAAAAC TA T T GAT

Non-tumor

\section{MAnMMAnMM}
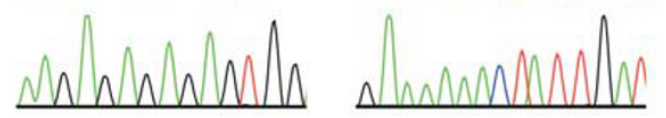

C

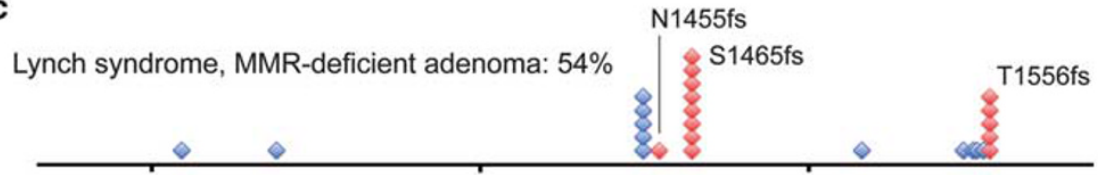

Lynch syndrome, MMR-proficient adenoma: $8 \%$

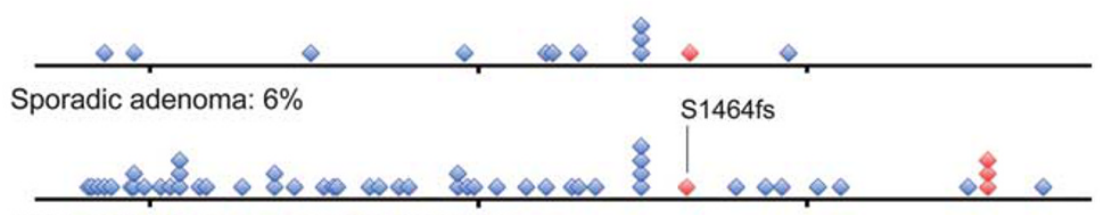

Lynch syndrome, adenocarcinoma: $55 \%$

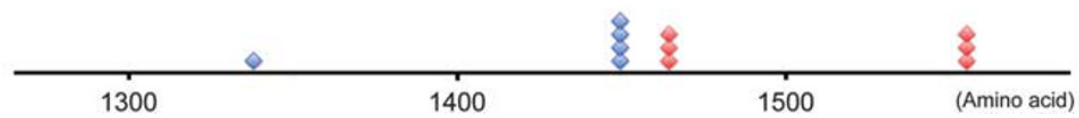

Figure 3 Mismatch repair (MMR)-deficient adenomas in patients with Lynch syndrome frequently show frameshift mutations of $R N F 43$ and $A P C$. (a) Sanger sequencing results showing frameshift $R N F 43$ mutations affecting repeat sequences (underlined). (b) Frameshift $A P C$ mutations affecting repeat sequences within the mutation cluster region (underlined). (c) Distribution of protein-truncating $A P C$ mutations in the mutation cluster region. Prevalence of frameshift mutations affecting repeat sequences is indicated. Red markers indicate frameshift mutations affecting repeat sequences.

MMR-deficient adenomas suggest that biallelic MMR gene inactivation commonly precedes adenoma formation in these lesions. It is still conceivable that MMR-proficient adenomas acquire MMR deficiency before progression to adenocarcinomas. However, the $A P C$ mutation profile of Lynch syndrome-associated adenocarcinomas, simulating that of MMR-deficient adenomas, indicates that carcinogenesis via MMRproficient adenomas is not a major pathway. In addition, a previous study showed that focal loss of MMR proteins is rarely observed in Lynch syndromeassociated adenomas, ${ }^{10}$ further implying that the transition from an MMR-proficient to MMR-deficient adenoma is an uncommon event.
Recent studies identified MMR-deficient crypt foci as a potential precursor of colorectal cancers in patients with Lynch syndrome. ${ }^{11,28}$ These lesions are predominantly mono- or oligocryptic and lack adenomatous morphology, suggesting that they represent the earliest lesion in the Lynch syndrome-associated colorectal tumorigenesis. Obviously, MMR-deficient crypt foci do not give rise to MMR-proficient adenomas; therefore, these lesions are related to distinct tumorigenic pathways. Moreover, it is unclear what proportions of adenocarcinomas in Lynch syndrome are derived from MMR-deficient foci. However, considering that the majority of the Lynch syndromeassociated adenocarcinomas acquire MMR-deficiency 


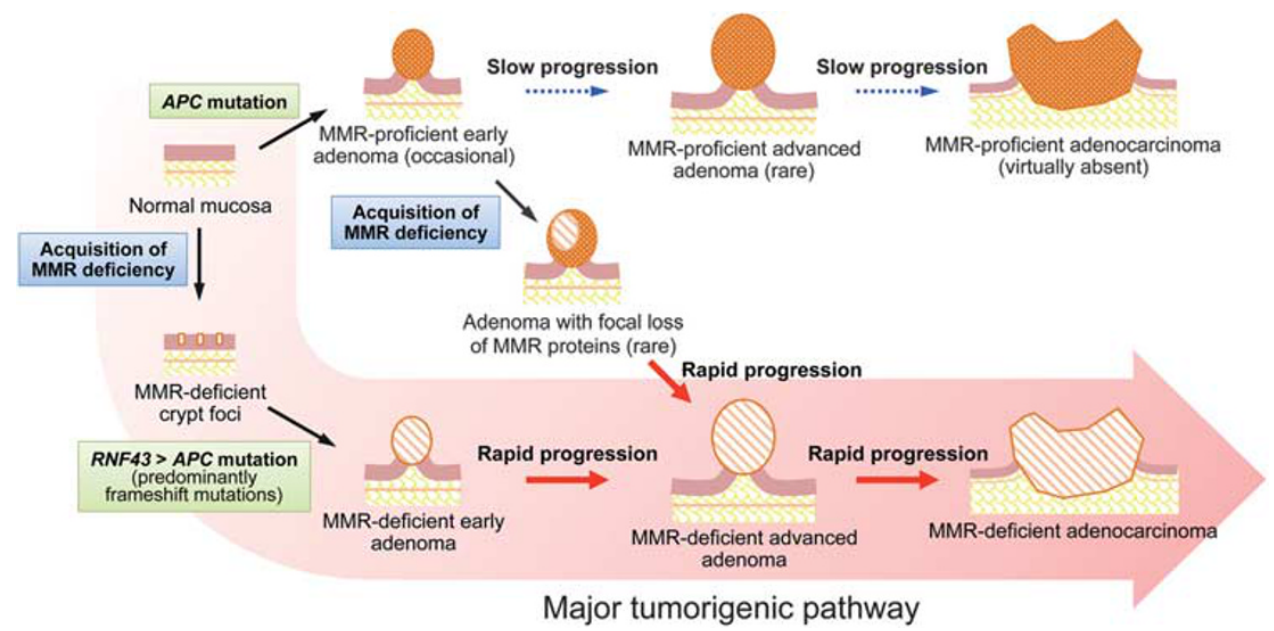

Figure 4 Model of Lynch syndrome-associated colorectal tumorigenesis. A subset of early colorectal adenomas in patients with Lynch syndrome are mismatch repair (MMR)-proficient, whereas most advanced adenomas are MMR-deficient, leading to the suggestion that acquisition of MMR deficiency occurs after adenoma formation. However, adenomas with focal loss of MMR proteins are rare and mutation profiles of RNF43 and APC in MMR-deficient and -proficient adenomas are highly different from each other; thus, transition from MMR-proficient to -deficient adenoma is unlikely to be a common phenomenon. Importantly, RNF43 and $A P C$ mutations in MMRdeficient adenomas are predominantly frameshift mutations affecting repeat sequences, implying that MMR-deficiency usually precedes the adenoma formation and that MMR-deficient crypt foci are major precursors.

before adenoma formation, MMR-deficient crypt foci may be the major precursor of Lynch syndromeassociated colorectal cancers (Figure 4).

In sporadic colorectal polyps and cancers, RNF43 mutations show strong mutual exclusivity to other WNT pathway gene mutations..$^{20,25}$ However, significant proportions of RNF43-mutated adenomas and adenocarcinomas had concurrent $A P C$ mutations in patients with Lynch syndrome. RNF43 and $A P C$ mutations activate the WNT signaling pathway through distinct mechanisms. RNF43 antagonize WNT signaling by targeting WNT receptors for proteasomal degradation; consequently, RNF43 mutations enhance liganddependent pathway activation. ${ }^{21}$ In contrast, APC inactivation constitutively stabilizes $\beta$-catenin and upregulates the WNT pathway in a cell autonomous and ligand-independent manner. ${ }^{29}$ Thus, although it remains elusive why $R N F 43$ and $A P C$ mutations often coexisted only in Lynch syndrome-associated colorectal tumors, theoretically, the acquisition of $A P C$ mutations might further activate WNT signaling even in the presence of RNF43 mutations.

This study revealed the frequent presence of RNF43 mutations and a unique $A P C$ mutation profile, which are likely consequences of MMR deficiency, in Lynch syndrome-associated MMRdeficient adenomas and adenocarcinomas. The characteristic mutation profiles of WNT pathway genes suggest that MMR deficiency is commonly acquired before adenoma formation during tumorigenesis. This further implies that Lynch syndromeassociated colorectal tumors already have a considerable mutation burden at the time of adenoma formation, potentially providing an additional explanation for their rapid progression.

\section{Acknowledgments}

We thank Ms Sachiko Miura, Ms Toshiko Sakaguchi, and Ms Chizu Kina for their skilful technical assistance and Dr Chiaki Inagaki for case selection.

\section{Disclosure/conflict of interest}

The authors declare no conflict of interest.

\section{References}

1 Vasen HF. Review article: the Lynch syndrome (hereditary nonpolyposis colorectal cancer). Aliment Pharmacol Ther 2007;26:113-126.

2 Lynch HT, Snyder CL, Shaw TG, et al. Milestones of Lynch syndrome: 1895-2015. Nat Rev Cancer 2015;15: 181-194.

3 Barrow E, Hill J, Evans DG. Cancer risk in Lynch Syndrome. Fam Cancer 2013;12:229-240.

4 Bonadona V, Bonaiti B, Olschwang S, et al. Cancer risks associated with germline mutations in MLH1, MSH2, and MSH6 genes in Lynch syndrome. JAMA 2011;305: 2304-2310.

5 Loukola A, Salovaara R, Kristo P, et al. Microsatellite instability in adenomas as a marker for hereditary nonpolyposis colorectal cancer. Am J Pathol 1999;155: 1849-1853.

6 De Jong AE, Morreau H, Van Puijenbroek M, et al. The role of mismatch repair gene defects in the development of adenomas in patients with HNPCC. Gastroenterology 2004;126:42-48.

7 Pino MS, Mino-Kenudson M, Wildemore BM, et al. Deficient DNA mismatch repair is common in Lynch syndrome-associated colorectal adenomas. J Mol Diagn 2009;11:238-247. 
8 Walsh MD, Buchanan DD, Pearson SA, et al. Immunohistochemical testing of conventional adenomas for loss of expression of mismatch repair proteins in Lynch syndrome mutation carriers: a case series from the Australasian site of the colon cancer family registry. Mod Pathol 2012;25:722-730.

9 Yurgelun MB, Goel A, Hornick JL, et al. Microsatellite instability and DNA mismatch repair protein deficiency in Lynch syndrome colorectal polyps. Cancer Prev Res 2012;5:574-582.

10 Tanaka M, Nakajima T, Sugano K, et al. Mismatch repair deficiency in Lynch syndrome-associated colorectal adenomas is more prevalent in older patients. Histopathology 2016;69:322-328.

11 Kloor M, Huth C, Voigt AY, et al. Prevalence of mismatch repair-deficient crypt foci in Lynch syndrome: a pathological study. Lancet Oncol 2012;13: 598-606.

12 Cancer Genome Atlas N. Comprehensive molecular characterization of human colon and rectal cancer. Nature 2012;487:330-337.

13 Seshagiri S, Stawiski EW, Durinck S, et al. Recurrent R-spondin fusions in colon cancer. Nature 2012;488: 660-664.

14 Giannakis $\mathrm{M}, \mathrm{Mu} \mathrm{XJ}$, Shukla SA, et al. Genomic correlates of immune-cell infiltrates in colorectal carcinoma. Cell Rep 2016;17:1206.

15 Shibata H, Toyama K, Shioya H, et al. Rapid colorectal adenoma formation initiated by conditional targeting of the Apc gene. Science 1997;278:120-123.

16 Harada $\mathrm{N}$, Tamai $\mathrm{Y}$, Ishikawa $\mathrm{T}$, et al. Intestinal polyposis in mice with a dominant stable mutation of the beta-catenin gene. EMBO J 1999;18:5931-5942.

17 Lamlum H, Papadopoulou A, Ilyas M, et al. APC mutations are sufficient for the growth of early colorectal adenomas. Proc Natl Acad Sci USA 2000;97: 2225-2228

18 Borras E, San Lucas FA, Chang K, et al. Genomic landscape of colorectal mucosa and adenomas. Cancer Prev Res 2016;9:417-427.

19 Miyaki M, Iijima T, Kimura J, et al. Frequent mutation of beta-catenin and APC genes in primary colorectal tumors from patients with hereditary nonpolyposis colorectal cancer. Cancer Res 1999;59:4506-4509.

20 Giannakis M, Hodis E, Jasmine Mu X, et al. RNF43 is frequently mutated in colorectal and endometrial cancers. Nat Genet 2014;46:1264-1266.

21 Koo BK, Spit M, Jordens I, et al. Tumour suppressor RNF43 is a stem-cell E3 ligase that induces endocytosis of Wnt receptors. Nature 2012;488:665-669.

22 Thompson BA, Spurdle AB, Plazzer JP, et al. Application of a 5-tiered scheme for standardized classification of 2,360 unique mismatch repair gene variants in the InSiGHT locus-specific database. Nat Genet 2014;46: 107-115.

23 Suraweera N, Duval A, Reperant M, et al. Evaluation of tumor microsatellite instability using five quasimonomorphic mononucleotide repeats and pentaplex PCR. Gastroenterology 2002;123:1804-1811.

24 Miya M, Sato Y, Fukunaga T, et al. MiFish, a set of universal PCR primers for metabarcoding environmental DNA from fishes: detection of more than 230 subtropical marine species. R Soc Open Sci 2015;2: 150088.

25 Sekine S, Yamashita S, Tanabe T, et al. Frequent PTPRK-RSPO3 fusions and RNF43 mutations in colorectal traditional serrated adenoma. J Pathol 2016;239: 133-138.

26 Christie M, Jorissen RN, Mouradov D, et al. Different APC genotypes in proximal and distal sporadic colorectal cancers suggest distinct WNT/beta-catenin signalling thresholds for tumourigenesis. Oncogene 2013;32: 4675-4682.

27 Albuquerque C, Bakker ER, van Veelen W, et al. Colorectal cancers choosing sides. Biochim Biophys Acta 2011;1816:219-231.

28 Staffa L, Echterdiek F, Nelius N, et al. Mismatch repairdeficient crypt foci in Lynch syndrome-molecular alterations and association with clinical parameters. PLoS ONE 2015;10:e0121980.

29 Clevers H, Nusse R. Wnt/beta-catenin signaling and disease. Cell 2012;149:1192-1205.

Supplementary Information accompanies the paper on Modern Pathology website (http://www.nature.com/ modpathol) 